\title{
Effects of Casein Kinase 2 Alpha 1 Gene Expression on Mice Liver Susceptible to Type 2 Diabetes Mellitus and Obesity
}

\author{
Yu-Ching Lan ${ }^{1 \#, ~ Y e h-H a n ~ W a n g 2 \#, ~ H s i n-H a n ~ C h e n ~}{ }^{3}$, Sui-Foon Lo ${ }^{4}$, Shih-Yin Chen ${ }^{4,5}$ and Fuu-Jen Tsai ${ }^{4,5,6}{ }^{\bowtie}$ \\ 1. Department of Health Risk Management, China Medical University, 40402 Taichung, Taiwan; \\ 2. Department of Anatomical Pathology, Taipei Institute of Pathology, School of Medicine, National Yang-Ming University , 11221 Taipei, Taiwan; \\ 3. Division of Plastic and Reconstructive Surgery, China Medical University Hospital, 40402 Taichung, Taiwan; \\ 4. School of Chinese Medicine, China Medical University, 40402 Taichung, Taiwan; \\ 5. Genetics Center, Medical Research, China Medical University Hospital, 40447 Taichung, Taiwan; \\ 6. Department of Medical Genetics, China Medical University Hospital, 40447 Taichung, Taiwan, R.O.C. \\ \# YCL and YHW contributed equally to this work and are co-first authors to this article. \\ $\square$ Corresponding author: chenshihy@gmail.com (SYC); d0704@mail.cmuh.org.tw (FJT), Genetics Center, Medical Research, China Medical University Hospital, \\ No. 2, Yuh-Der Road, 40447 Taichung, Taiwan, R.O.C.
}

(C) The author(s). This is an open access article distributed under the terms of the Creative Commons Attribution License (https://creativecommons.org/licenses/by/4.0/). See http:/ /ivyspring.com/terms for full terms and conditions.

Received: 2019.05.29; Accepted: 2019.09.18; Published: 2020.01.01

\begin{abstract}
Diabetes mellitus (DM) is a chronic disease found worldwide. Notably, BKS.Cg- Dock $7^{m}+/+$ Lepr $^{\mathrm{db}} / \mathrm{JNarl}$ mice are useful animal models for studying type 2 diabetes mellitus (T2DM). In this study, we investigated casein kinase 2 alpha 1 (CSNK2A1) gene and protein expression in the liver tissues of mice at different ages $(4,16$, and 32 weeks) using real-time quantitative polymerase chain reactions, western blotting, immunohistochemistry, and enzyme-linked immunosorbent assay. Our data paved the way for exploring BKS.Cg- Dock $7^{m}+/+$ Lepr $^{\mathrm{db}} / J \mathrm{Narl}$ in the mouse model by demonstrating a significant increase in gene and protein expression in T2DM $\left(+\operatorname{Lepr}^{\mathrm{db}} /+\operatorname{Lepr}^{\mathrm{db}}\right.$ ) mouse liver when compared to control (+Dock $7^{\mathrm{m}} /+$ Dock $7^{\mathrm{m}}$ ) mouse liver. We also observed that CSNK2A1 protein level in the serum of T2DM patient group was higher than that of the control group, although the data was not statistically significant. Based on our findings, we can now understand the role of CSNK2AI gene upregulation when encountering T2DM pathologies.
\end{abstract}

Key words: +Lepr ${ }^{\mathrm{db}} /+$ Lepr $^{\mathrm{db}}$ mice; T2DM; Casein Kinase 2 Alpha 1 (CSNK2A1).

\section{Introduction}

The protein kinase casein kinase 2 (CSNK2 or CK2) is a serine/threonine protein kinase that is expressed in most cell types [1]. CSNK2 phosphorylates various proteins that deal with cell cycle regulation, cell survival, cell morphology, cell metabolism, tumorigenesis, and cancer cell invasiveness [2]. It is composed of 2 large catalytic subunits, CK2a (44 kDa) and CK2 $\mathrm{a}^{\prime}(36 \mathrm{kDa})$, and 2 small non-catalytic CK2 $\beta$ subunits $(25 \mathrm{kDa})$. CK2 $\alpha$ is a human enzyme encoded by the CSNK2A1 gene [3]. Notably, CSNK2A1 has been found to be highly expressed in a wide variety of cancers. At the transcriptional and/or protein level, CK2 overexpression, particularly in the a catalytic subunit (CK2a or CSNK2A1), has been observed in many cancers ${ }^{[4]}$. A growing body of evidence shows that the insulin signaling system plays a key role in cancer development and progression. CK2 also plays an important role in the regulation of carbohydrate metabolism [5]. Hence, CK2 has recently been recognized as a "master kinase" that is involved in many important cellular processes by controlling the activity of several other kinases [1].

$\mathrm{G}$ protein-coupled receptors (GPCRs) regulate the enzymatic activity of virtually all cell types, including pancreatic $\beta$-cells. Furthermore, $\beta$-cell M3 
muscarinic receptors (M3Rs) play an important role in maintaining proper whole-body glucose homeostasis [6]. Similar to other GPCRs, various kinases modulate M3R activity via phosphorylation. When insulin is released, CK2-dependent phosphorylation of $\beta$-cell M3Rs significantly damages M3R-mediated increases in protein expression. The physiological relation between CK2 phosphorylation and GPCRs suggests that the kinases acting on $\beta$-cell GPCRs may be considered as targets for therapy. CK2 inhibition has also been shown to strongly enhance M3R-stimulated insulin secretion in isolated pancreatic islets or cultured $\beta$-cells [7]. However, studies about CSNK2A1 expression in liver cells are severely lacking.

Diabetes mellitus (DM) is a complex disease that occurs due to absolute or relative insulin deficiency. Lifestyle modifications and nutritional adjustments are some of the best ways to prevent and treat type 2 diabetes mellitus (T2DM) ${ }^{[8]}$. Notably, genetic factors have also been shown to play an important role in DM and have been suggested as new ways of combating T2DM [8,9]. In this study, we show that CSNK2A1 is involved in the regulation of glucose stimulated insulin secretion (GSIS). A previous study demonstrated that increased CSNK2A1 activity is correlated with enhanced insulin secretion and consistent with the MS-based proteomic profile, a significant reduction of CK2A levels was observed in NOD (non-obese diabetic mice) diabetic islets [10]. In previous cancer studies, CSNK2A1 overexpression at the transcriptional and/or protein level was observed in breast and liver cancer samples [4,11,12]. Due to a lack of studies regarding CSNK2A1 expression at the genomic and protein levels in T2DM and obesity, we used the T2DM and obesity mouse model in this study to investigate the impact of CSNK2A1 on T2DM and obesity. Here, we showed strong evidence of the role of CSNK2A1 gene and protein expression in obesity and T2DM. By using an animal model, we demonstrated CSNK2A1 gene and protein expression in the liver tissue of different mouse groups to better understand the role in the pathological features of T2DM.

\section{Materials and methods}

\section{Animal model}

Twenty-four 4-week-old male BKS.Cg- Dock7m $+/+$ Leprdb/JNarl mice along with control $(+$ Dock7m/+Dock7m; $\mathrm{n}=12)$ and T2DM $\left(+\right.$ Lepr $^{\mathrm{db}} /+$ Lepr $\left.^{\mathrm{db}} ; \mathrm{n}=12\right)$ mice were obtained from the National Laboratory Animal Center (NLAC) in Taiwan. All animals were raised in individual cages and placed in rooms that had a relative humidity of $50-70 \%$, constant temperature of $22-25^{\circ} \mathrm{C}$, and $12 \mathrm{~h}$ light/dark cycles. There were 6 groups in the study: 3 control groups at 4, 16, and 32 weeks; and 3 T2DM groups at 4, 16, and 32 weeks. Our study was reviewed and approved by the Institutional Animal Care and Use Committee (IACUC) of China Medical University (IACUC: 2016-221).

\section{Real-time quantitative polymerase chain reaction}

The RNeasy Mini Kit (Qiagen, Germantown, MD, USA) was used to isolate total RNA from ground liver tissue of control and T2DM mice. The SuperScript First-Strand Synthesis Kit (Invitrogen) was used for cDNA transcription. To study gene expression, real-time quantitative polymerase chain reactions (RT-qPCRs) were performed using TaqMan assays (Applied Biosystems, CA, USA) for murine CSNK2A1 (NM_007788.3) on a Prism 7900HT Sequence Detection System (Applied Biosystems). The target gene expression levels were normalized to mice glyceraldehyde-3-phosphate dehydrogenase (GAPDH; M32599).

\section{Western blot analysis}

In this study, murine anti-CSNK2A1 (GTX107897) monoclonal antibodies were used to detect CSNK2A1 via western blotting procedures described previously [13]. Briefly, frozen liver tissue samples were homogenized with 3 volumes of $10 \mathrm{mM}$ ice-cold phosphate buffer ( $\mathrm{pH} 7.0$ ) containing $1 \mathrm{mM}$ EDTA, $0.25 \mathrm{M}$ sucrose, $1 \mathrm{mM}$ sodium azide, and 0.1 $\mathrm{mM}$ phenylmethylsulfonyl fluoride. Samples were then centrifugated at $20,000 \times g$ for $30 \mathrm{~min}$ at $4{ }^{\circ} \mathrm{C}$. Protein concentrations were measured using BCA assay (Pierce Biotechnology, Rockford, IL, USA) with albumin as the standard. The tissue lysates were subjected to denaturing electrophoresis via $10 \%$ SDS-polyacrylamide gel, electro-transferred to PVDF membranes, and immune-stained with CSNK2A1 and $\beta$-actin antibodies. The bands were analyzed using the Enhanced Chemiluminescence Kit (Amersham, Buckinghamshire, UK).

\section{Immunohistochemistry analysis}

CSNK2A1 protein expression was determined via immunohistochemistry (IHC) analysis using paraffin-embedded liver sections. Anti-CSNK2A1 IHC staining was carried out using the LsAB Kit (DAKO, Glostrup, Denmark). All tissue sections were de-waxed, treated with Proteinase K enzyme, and the endogenous peroxidase activity was blocked by incubating with 3\% hydrogen peroxide for $10 \mathrm{~min}$. After washing with phosphate-buffered saline (PBS; $\mathrm{pH}$ 7.6) for $5 \mathrm{~min}$, the slides were incubated with anti-CSNK2A1 antibodies (GTX84369; GeneTex, 
Hsinchu, Taiwan) for $30 \mathrm{~min}$ at $37^{\circ} \mathrm{C}$, followed by rabbit anti-rat antibodies and a goat anti-rabbit HRP polymer for $15 \mathrm{~min}$. The immunocomplexes were visualized using DAB solution (DAKO) for $5 \mathrm{~min}$. Samples were washed with PBS $(\mathrm{pH} 7.6)$ in order to perform all the necessary steps $[14,15,16]$.

\section{Enzyme linked immunosorbent assay (ELISA) of CSNK2A1 protein levels in mouse liver and human serum}

Mouse liver tissue were minced after weighing and homogenized in PBS with a glass homogenizer on ice. The homogenates were then centrifuged at 5,000 $\times g$ for $5 \mathrm{~min}$ and supernatants were collected to measure protein concentrations at room temperature. CSNK2A1 protein levels in mouse liver and human serum were determined using a suite of commercial kits (Catalogue No: EM7856 for mouse and EH1138 for human; Wuhan Fine Biological Technology Co., Hubei, China) according to the manufacturer's instructions.

\section{Biochemical assessment}

Glutamic-pyruvic transaminase (GPT) levels were determined using a Spotchem EZ analyzer that uses carrier strips (Menarini Diagnostics, Wokingham, UK). Samples were run on the instrument using previously described standard methodologies [17]. Analyses and operations were based on the manufacturer's standard processes and all samples were run by the same technician.

\section{Patients and sample collection}

In this study, 2 male and 6 female patients (ranging from 45-65 years old) that fulfilled the diagnostic criteria of obesity (BMI > 27) and T2DM were enrolled at the China Medical University Hospital in Taiwan between Aug 2014 and July 2015. Age- and gender-matched unrelated healthy controls were also obtained from the general population at the same hospital during the same time period. Serum samples were collected for ELISA. The protocols were approved by the ethical committee of China Medical University Hospital (No. CMUH103-REC2-071). Informed consent was obtained from all individuals enrolled in the study.

\section{Statistical analysis}

Data is expressed as mean \pm standard error of 3 independent experiments. Statistical comparison between the test and control groups was performed using the Student's $t$-test. $\mathrm{P}<0.05$ was considered to be statistically significant.

\section{Results}

Figure 1A shows the body weights of the control $\left(+\right.$ Dock7m/+Dock7m) and T2DM (+Lepr ${ }^{\mathrm{db}} /+$ Lepr $\left.^{\mathrm{db}}\right)$ mouse models over 4,16 , and 32 weeks. Our data indicated that the body weights in the T2DM mouse groups increased significantly when compared to the control groups, thus doubling up as an obesity animal model $(\mathrm{P}<0.05)$. We also observed that blood glucose levels in the T2DM mouse groups increased significantly from 4 to 32 weeks $(\mathrm{P}<0.05)$ i.e., more than $500 \mathrm{mg} / \mathrm{dL}$ of blood glucose was detected in the T2DM mouse groups at 32 weeks (Figure 1B).

Control (+Dock7m/+Dock7m) and T2DM $\left(+\right.$ Lepr $^{\mathrm{db}} /+$ Lepr $\left.^{\mathrm{db}}\right)$ mice were individually sacrificed at 4,16 , and 32 weeks. RNA from the liver tissues was then extracted for quantitative real-time reverse transcription polymerase chain reaction analysis. The qPCR data in Figure 2 shows CSNK2A1 gene expression in the liver tissue of mice aged 4, 16, and 32 weeks. The results showed that CSNK2A1 gene expression in the liver tissue of + Lepr $^{\mathrm{db}} /+$ Lepr $^{\mathrm{db}}$ mice (T2DM) was significantly higher than that in the liver tissue of + Dock7m $/+$ Dock $7 \mathrm{~m}$ mice (control) $(\mathrm{P}<0.05$; Figure 2). Therefore, the results suggest upregulated CSNK2A1 gene expression in T2DM mice.

Control and T2DM mice were individually sacrificed at 4, 16, and 32 weeks. Mouse liver tissues were homogenized and $20 \mu \mathrm{g}$ of protein was analyzed via western blotting using anti-CSNK2A1 and $\beta$-actin antibodies. Figure $3 \mathrm{~A}$ shows representative blots for control (lanes 1, 3, and 5) and T2DM (lanes 2, 4, and 6) mice at 4,16 , and 32 weeks, respectively. Liver tissues were also excised, fixed, embedded, and sectioned for IHC staining. Figure 3B shows IHC-processed CSNK2A1 protein expression in the liver tissues of mice aged 4, 16, and 32 weeks. The results showed that CSNK2A1 protein expression in the liver tissue of + Lepr $^{\mathrm{db}} /+$ Lepr $^{\mathrm{db}}$ mice (T2DM) was significantly higher than that in the liver tissue of + Dock $7 \mathrm{~m} /+$ Dock $7 \mathrm{~m}$ mice (control) (Figure 3). Therefore, the results suggest upregulated CSNK2A1 protein expression in T2DM mice.

Control and T2DM mice were individually sacrificed at 4, 16, and 32 weeks. Proteins from the liver tissues were then extracted for ELISA. The ELISA data in Figure 4 shows CSNK2A1 protein levels in the liver tissue of mice aged 4, 16, and 32 weeks. The results showed that CSNK2A1 protein levels in the liver tissue of + Leprdb$^{\mathrm{db}} /+$ Leprdb $^{\mathrm{db}}$ mice (T2DM) were significantly higher than that in the liver tissue of + Dock7m/ +Dock $7 \mathrm{~m}$ mice (control) at 16 and 32 weeks $(\mathrm{P}<0.05$; Figure 4$)$. Therefore, the results suggest upregulated CSNK2A1 protein levels in T2DM mice.

Next, mice were sacrificed and blood samples 
were collected from each mouse in order to measure serum glutamic pyruvic acid transaminase (GPT) activity using a Spotchem EZ analyzer that uses carrier strips. Our data indicated that high blood glucose levels in the T2DM group (+Lepr ${ }^{\mathrm{db}} /+$ Lepr $^{\mathrm{db}}$ ) significantly increased serum GPT activity when compared to the control group $(+$ Dock $7 \mathrm{~m} /+$ Dock $7 \mathrm{~m})$ $(\mathrm{P}<0.05$; Figure 5).

Finally, ELISA was used to detect CSNK2A1 expression in T2DM patients with obesity. Serum samples were collected from $8 \mathrm{~T} 2 \mathrm{DM}$ patients and 3 non-T2DM patients (BMI > 27). The ELISA data in Figure 6 shows CSNK2A1 protein levels in human serum. The results showed that CSNK2A1 protein levels in the serum of the T2DM patient group was higher than that in the serum of the control group, although the data was not statistically significant (Figure 6). Therefore, the results suggest upregulated CSNK2A1 protein levels in T2DM patients.

\section{Discussion}

To our knowledge, this is the first systematic study that used the time serial animal model spanning the early to the late DM state to investigate the association between CSNK2A1, obesity, and T2DM from the genomic to the phenotypic level in the liver. Our animal model results generally demonstrated diabetes and obesity in animals by showing a significant increase in body weight and blood glucose levels in the T2DM mouse group [18,19]. Furthermore, biochemical assessment of GPT in T2DM mice also showed a significant increase over time even though its level was significantly high in the control group, which showed low liver functionality in the T2DM mouse group [20,21].

In this study, we investigated CSNK2A1 gene and protein expression in the liver tissues of mice at different ages $(4,16$, and 32 weeks) via a systematic method using real-time qPCRs, western blot assays, IHC, and ELISA. Our data demonstrated a significant increase in gene and protein expression in the liver tissues of T2DM (+Lepr ${ }^{\mathrm{db}} /+$ Lepr $\left.^{\mathrm{db}}\right)$ and control $(+$ Dock $7 \mathrm{~m} /+$ Dock $7 \mathrm{~m})$ mice. We also observed similar results in the serum samples of T2DM patients with obesity; however, the interpretation was limited by the small sample size used in this study. Hence, further studies are required to explore CSNK2A1 protein levels in T2DM patients with obesity using a larger sample size. However, our data paved the way to exploring the BKS.Cg- Dock7m $+/+$ Lepr $^{\mathrm{db}} / \mathrm{JNarl}$ mouse model. Using our results, we can now better understand the role of CSNK2A1 gene expression in T2DM pathological features.

A)

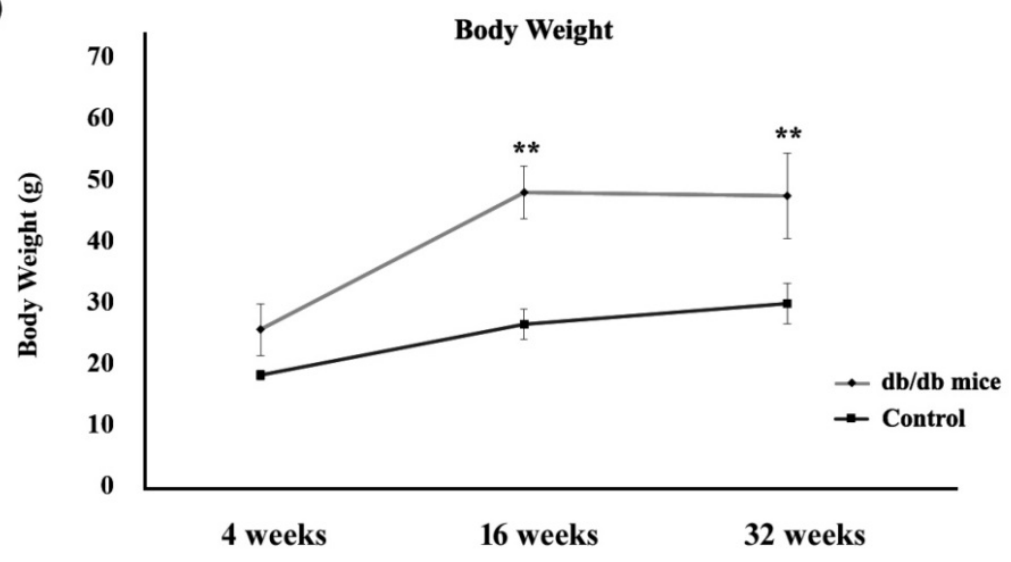

B)

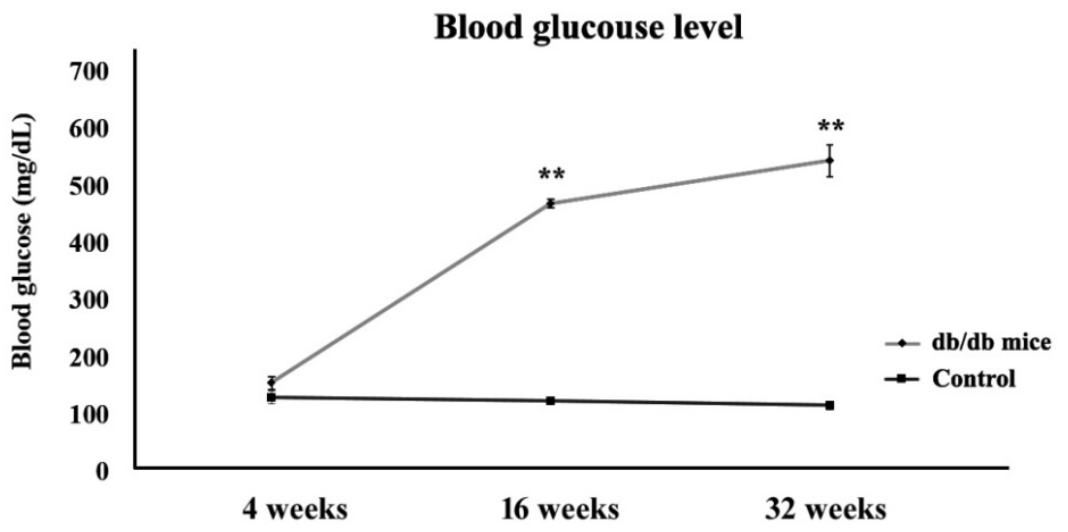


Figure 1. Estimation of (A) body weight and (B) blood glucose levels in control $(\square)$ and T2DM $(\bullet)$ mouse models $(* *, P<0.05)$.

\section{CSNK2A1}

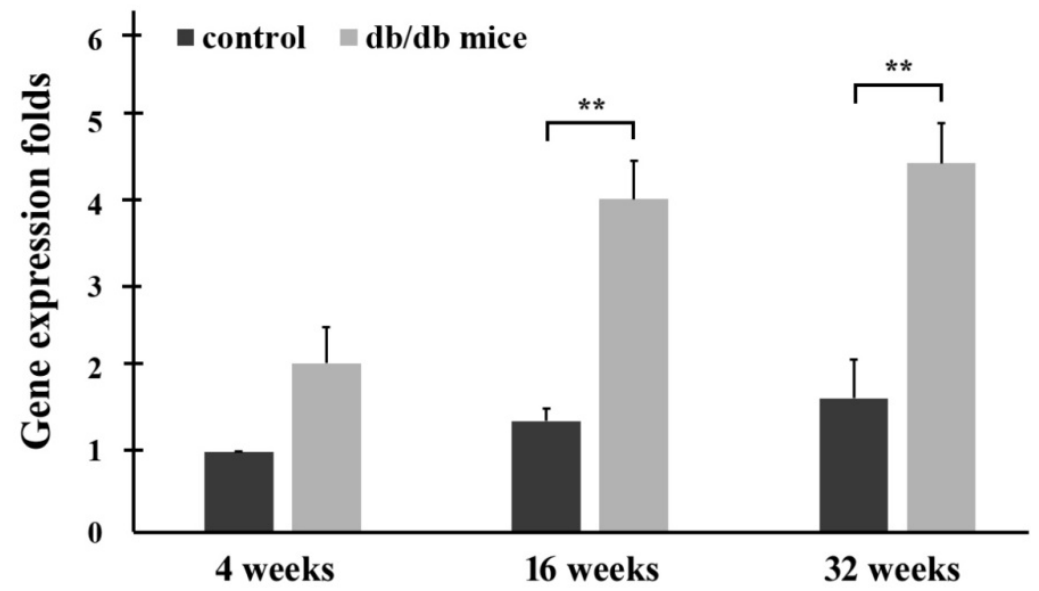

Figure 2. Quantitative real-time reverse transcription polymerase chain reaction analysis of the CSNK2Al gene in the liver tissues of control (+Dock7m/+Dock7m) and T2DM (+Leprdb/+Lepr ${ }^{\mathrm{db}}$ ) mice at 4,16 , and 32 weeks. Gene expression data of CSNK2A1 was calculated after normalizing against GADPH (**, P < 0.05).

A)

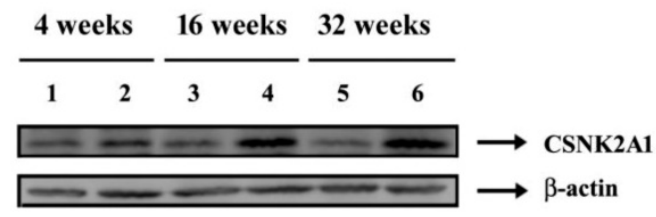

B)

+Dock7m /+Dock7m mice (control mice)

(60 X)

+Dock7m /+Dock7m mice (control mice)

(150 X)
+Leprdb / +Leprdb mice (T2D mice)

(60 X)

+Leprdb / +Leprdb mice (T2D mice)

(150 X)

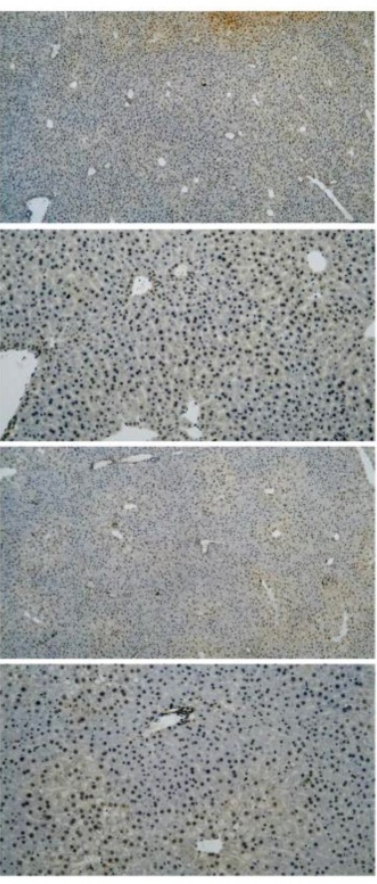

4 weeks
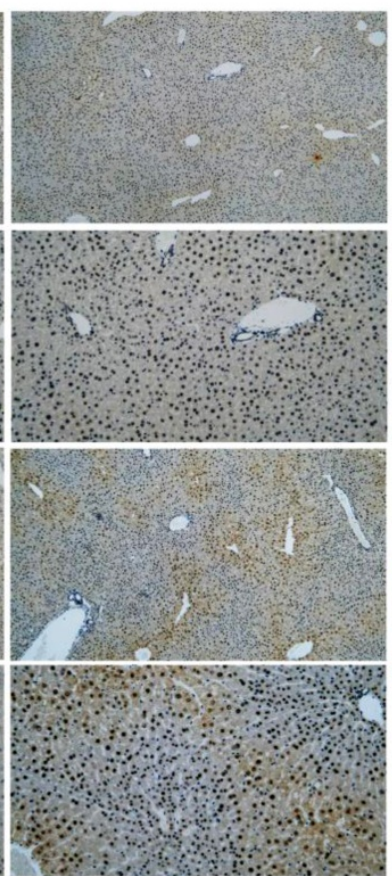

16 weeks

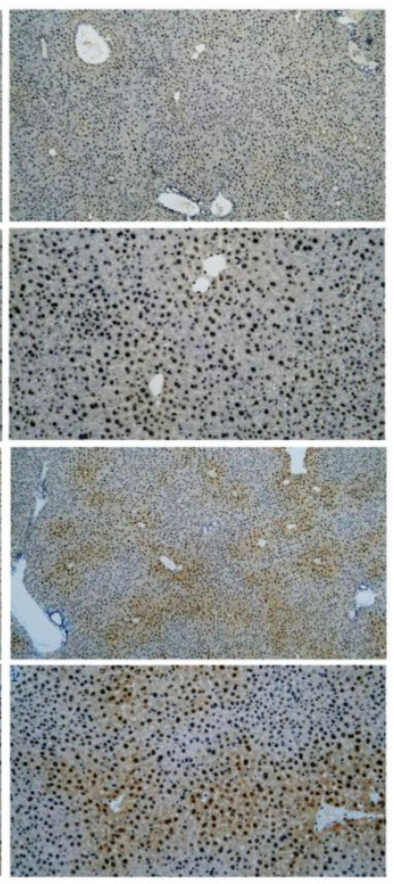

32 weeks

Figure 3. (A) Western blot analyses of CSNK2A1 protein expression in the liver tissues of control (+Dock7m/+Dock7m; lanes 1 , 3, and 5) and T2DM (+Leprdb/+Leprdb; lanes 2, 4, and 6) mice at 4, 16, and 32 weeks, respectively. (B) Representative IHC-processed CSNK2A1 expression in T2DM mice $(+$ Leprdb/+Leprdb) is shown. Progression of the mouse models for T2DM is also shown. Liver tissues were excised, fixed, embedded, and sectioned for IHC staining as described in the Materials and Methods. 


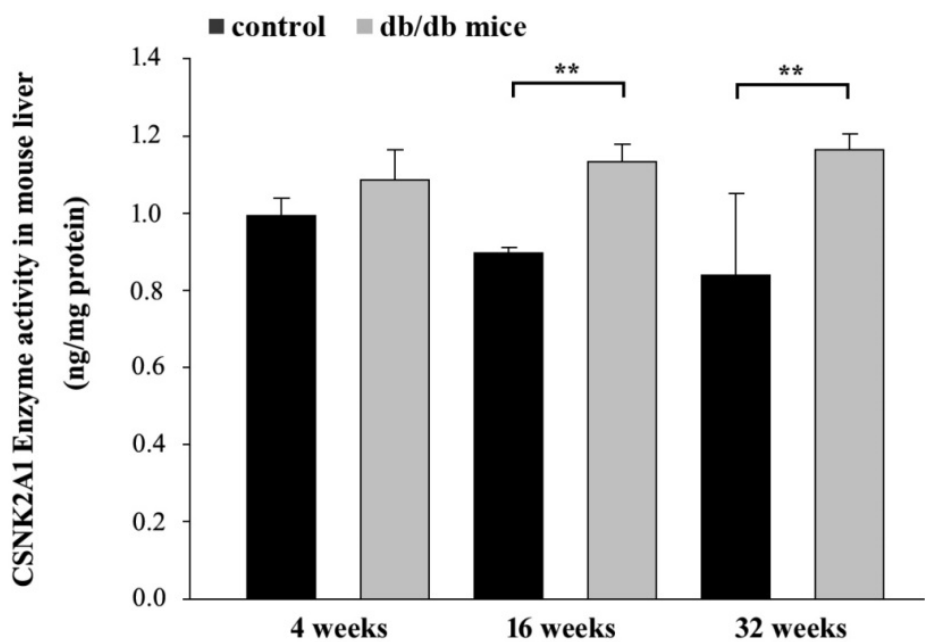

Figure 4. ELISA-mediated measurement of CSNK2Al protein levels in the liver tissues of control (+Dock7m/+Dock7m) and T2DM (+Leprdb/+Leprdb) mice at 4, 16, and 32 weeks. **, $\mathrm{P}<0.05$ for the indicated comparisons.

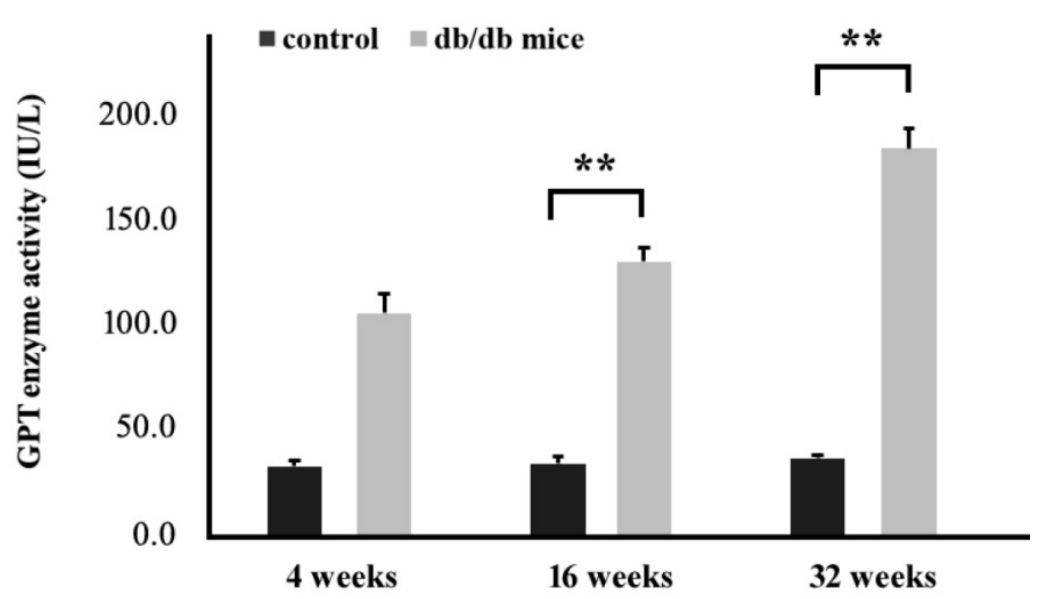

Figure 5. Measurement of GPT activity in the blood of control (+Dock7m/+Dock7m) and T2DM (+Leprdb/+Leprdb) mice at 4, 16 , and 32 weeks. **, P $<0.05$ for the indicated comparisons.

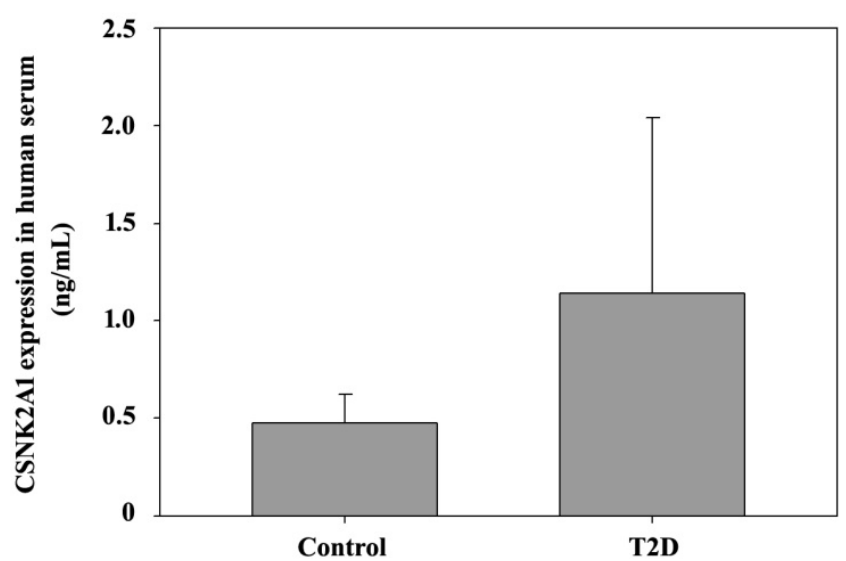

Figure 6. ELISA-mediated measurement of CSNK2AI protein levels in the human serum of T2DM patients and controls.

Notably, CSNK2A1 transcripts and proteins are reported to be upregulated in many kinds of cancers [4,22], such as multiple myeloma [23], breast cancer [11], and liver cancer [12]. Moreover, poor patient survival rates have been correlated with CSNK2A1 overexpression [24]. In addition, CSNK2A1 gene overexpression was observed in large HCC patient cohorts [25]. A study by Zhang et al. found that CSNK2A1 was significantly overexpressed (greater than a 2-fold increase) at the mRNA level in HCC tissues, the expression of which was also reflected at the CSNK2A1 protein level [12]. In this study, the strong expression of the CSNK2A1 gene in the liver tissues of T2DM mice not only showed the same results as the previously mentioned study, but also showed a high expression level in $\beta$-cells [7]. The CSNK2A1 gene was further observed to be expressed at a 1.2-fold higher value in human $\beta$-cells isolated from T2DM patients when compared to non-diabetic individuals [26]. Thus, these results provide sufficient evidence to associate CSNK2A1 gene expression with T2DM. Furthermore, CSNK2A1 protein expression in the diabetic group was also significantly higher than the control group. CSNK2A1 inhibition in pancreatic $\beta$-cells, knockdown of CSNK2A1 expression, and/or genetic deletion of CSNK2A1 $\beta$-cells in mutant mice 
were previously shown to negatively regulate insulin secretion in vivo and in vitro [7]. An association between CSNK2A1 and the atypical NF-KB pathway was also confirmed previously [27]. Notably, insulin/IGF-1 acts via 2 mechanisms (AKT and mTOR signaling) to activate NF-kB ${ }^{[28]}$. Combining these findings with our results, it is possible that CSNK2A1 plays an important role in T2DM development. Therefore, the mechanisms of insulin sensitivity and glucose homeostasis warrant further investigation.

Notably, compared to our study that showed upregulated CSNK2A1 gene and protein expression, the previous study used a non-obese diabetic (NOD) mouse model that resulted in the downregulation of protein levels in the islet cells of the diabetic group when compared to the islet cells of the non-diabetic group [10]. The difference between our animal model (Obese Diabetes Mouse Model) and the Sacco animal model (Non-Obese Diabetes Model) resided in the fact that our model showed a possible correlation between CSNK2A1 and the obesity mechanism and regulation. Furthermore, we also presented time serial changes for obesity and diabetic parameters such as BMI, blood glucose, serum GPT enzyme activity, and CSNK2A1 gene and protein expression in this study. Previous studies showed that a significant increase in the level of serum GPT was noted in STZ-diabetic mice [29]. In addition, a high level of serum GPT was observed in diabetic mice with fatty liver as compared to the control [30]. Serum GPT is commonly used to detect non-alcoholic fatty liver disease and has been associated with increased risk of T2DM [31]. Therefore, all these evidences showed that the CSNK2A1 protein possibly played an important role in the obesity mechanism.

In conclusion, our study showed the upregulation of CSNK2A1 gene expression and protein expression in T2DM mice, which confirmed the relationship between CSNK2A1 and T2DM. Altogether, our results indicate that CSNK2A1 plays an important role in T2DM and obesity regulation. To our knowledge, this is the first study that used the time serial animal model to investigate the association between CSNK2A1 and obesity and T2DM disease progression from the gene expression level to the phenotypic level. However, further studies are required to understand the mechanisms of CSNK2A1 at the genomic and protein levels and to understand its association with pancreatic $\beta$-cell-mediated T2DM and obesity.

\section{Abbreviations}

T2DM: type 2 diabetes mellitus; CSNK2A1: casein kinase 2 alpha 1; qPCRs: quantitative polymerase chain reactions; IHC: immunohistochemistry; ELISA: enzyme-linked immunosorbent assay; GPT: glutamic-pyruvic transaminase; M3Rs: $\beta$-cell M3 muscarinic receptors; GAPDH: glyceraldehyde-3-phosphate dehydrogenase; PBS: phosphate buffered saline.

\section{Acknowledgements}

We are grateful to the patients for donating their samples to our research studies, staff within the research and clinical teams at Genetic Center, China Medical University Hospital for help in obtaining and processing samples for funding this research.

\section{Funding}

This work is supported by China Medical University Hospital in Taiwan (DMR-106-055 and DMR-106-116) in the design of the study and collection, analysis, and interpretation of data and in writing the manuscript.

\section{Availability of data and materials}

The datasets used and/or analyzed during the current study available from the corresponding author on reasonable request.

\section{Ethical approval and consent to participate}

The animal experiments were approved by the Institutional Animal Care and Use Committee of China Medical University (IACUC permit no. 2016-221). The human experiments were approved by the ethics committee/Institutional Review Board of China Medical University Hospital (no. CMUH103-REC2-071). All participants provided written informed consent.

\section{Author contributions}

Conception and design [SYC, YCL, YHW, FJT]; Analysis and interpretation of data [SYC, YCL, YHW, FJT, SFL, HHC]; Participated in drafting of the manuscript [SYC, YCL, YHW, FJT, HHC]; Critical revision of the manuscript for important intellectual content [SYC, YCL, YHW, FJT, HHC, SFL]. All authors read and approved the final manuscript.

\section{Competing Interests}

The authors have declared that no competing interest exists.

\section{References}

[1] St-Denis NA, Litchfield DW. Protein kinase CK2 in health and disease: From birth to death: the role of protein kinase CK2 in the regulation of cell proliferation and survival. Cell Mol Life Sci. 2009;66:1817-29.

[2] Bae JS, Park SH, Jamiyandorj U, et al. CK2alpha/CSNK2A1 Phosphorylates SIRT6 and Is Involved in the Progression of Breast Carcinoma and Predicts Shorter Survival of Diagnosed Patients. Am J Pathol. 2016;186:3297-315.

[3] Lozeman FJ, Litchfield DW, Piening C, et al. Krebs. Isolation and characterization of human cDNA clones encoding the alpha and the alpha' subunits of casein kinase II. Biochemistry. 1990;29:8436-47. 
[4] Ortega CE, Seidner Y, Dominguez I. Mining CK2 in cancer. PLoS One. 2014;9:e115609.

[5] Al Quobaili F, Montenarh M. CK2 and the regulation of the carbohydrate metabolism. Metabolism. 2012;61:1512-7.

[6] Ruiz de Azua I, Gautam D, Guettier JM, Wess J. Novel insights into the function of $\beta$-cell M3 muscarinic acetylcholine receptors: therapeutic implications. Trends Endocrinol Metab. 2011;22:74-80.

[7] Mario Rossi IRdA, Barella Luiz F, Sakamoto Wataru, et al. CK2 acts as a potent negative regulator of receptormediated insulin release in vitro and in vivo. Proc Natl Acad Sci U S A. 2015;112(49):E6818-24.

[8] Chen SY, Hsu YM, Lin YJ, et al. Current concepts regarding developmental mechanisms in diabetic retinopathy in Taiwan. Biomedicine (Taipei). 2016;6:7.

[9] Wang IX, Ramrattan G, Cheung VG. Genetic variation in insulin-induced kinase signaling. Mol Syst Biol. 2015;11:820.

[10] Sacco F, Humphrey SJ, Cox J, et al. Glucose-regulated and drug-perturbed phosphoproteome reveals molecular mechanisms controlling insulin secretion. Nature Communications. 2016;7.

[11] Giusiano S, Cochet C, Filhol O, et al. Protein kinase CK2alpha subunit over-expression correlates with metastatic risk in breast carcinomas: quantitative immunohistochemistry in tissue microarrays. Eur J Cancer. 2011:47:792-801.

[12] Zhang HX, Jiang SS, Zhang XF, et al. Protein kinase CK2alpha catalytic subunit is overexpressed and serves as an unfavorable prognostic marker in primary hepatocellular carcinoma. Oncotarget. 2015;6:34800-17.

[13] Lin CC, Chen KB, Tsai CH, Tsai FJ, Huang CY, Tang CH, Yang JS, Hsu YM, Peng SF, Chung JG. Casticin inhibits human prostate cancer DU 145 cell migration and invasion via Ras/Akt/NF-kB signaling pathways. J Food Biochem. 2019;43:e12902.

[14] Steneberg P, Bernardo L, Edfalk S, et al. The type 2 diabetes-associated gene ide is required for insulin secretion and suppression of alpha-synuclein levels in beta-cells. Diabetes. 2013;62:2004-14.

[15] Sekiguchi K, Kurabayashi M, Oyama Y, et al. Homeobox Protein Hex Induces SMemb/Nonmuscle Myosin Heavy Chain-B Gene Expression Through the cAMP-Responsive Element. Circulation Research 2001;88:52-8.

[16] Liu SC, Tsai CH, Wu TY, et al. Soya-cerebroside reduces IL-1 1 -induced MMP-1 production in chondrocytes and inhibits cartilage degradation: implications for the treatment of osteoarthritis. Food and Agricultural Immunology 2019;30:620-32.

[17] Hetzel N, Papasouliotis K, Dodkin S, Murphy K. Biochemical assessment of canine body cavity effusions using three bench-top analysers. J Small Anim Pract 2012;53:459-64.

[18] Chen H, Charlat O, Tartaglia LA, et al. Evidence that the diabetes gene encodes the leptin receptor: Identification of a mutation in the leptin receptor gene in $\mathrm{db} / \mathrm{db}$ mice. Cell 1996;84:491-5.

[19] Hummel KP, Dickie MM, Coleman DL. Diabetes, a new mutation in the mouse. Science. 1966;153:1127-8.

[20] Jadhao SB, Yang RZ, Lin Q, et al. Murine alanine aminotransferase: cDNA cloning, functional expression, and differential gene regulation in mouse fatty liver. Hepatology. 2004;39:1297-302.

[21] Hellman B, Larsson S, Westman S. Aspects of the glucose and amino acid metabolism in the liver and the diaphragm of normal and obese-hyperglycemic mice. Acta Physiol Scand. 1961;53:330-8.

[22] Chua MM, Ortega CE, Sheikh A, et al. CK2 in Cancer: Cellular and Biochemical Mechanisms and Potential Therapeutic Target, Pharmaceuticals (Basel). 2017;10(1).

[23] Piazza FA, Ruzzene M, Gurrieri C, et al. Multiple myeloma cell survival relies on high activity of protein kinase CK2. Blood. 2006;108:1698-707.

[24] Zhou B, Ritt DA, Morrison DK, et al. Protein Kinase CK2alpha Maintains Extracellular Signal-regulated Kinase (ERK) Activity in a CK2alpha Kinase-independent Manner to Promote Resistance to Inhibitors of RAF and MEK but Not ERK in BRAF Mutant Melanoma. J Biol Chem. 2016;291:17804-15.

[25] Kim HS, Chang YG, Bae HJ, et al. Oncogenic potential of CK2alpha and its regulatory role in EGF-induced HDAC2 expression in human liver cancer. FEBS J. 2014;281:851-61.

[26] Marselli L, Thorne J, Dahiya S, et al. Weir, Gene expression profiles of Beta-cell enriched tissue obtained by laser capture microdissection from subjects with type 2 diabetes. PLoS One. 2010;5:e11499.

[27] Bell L, Chowdhary R, Liu JS, et al. Integrated bio-entity network: a system for biological knowledge discovery. PLoS One. 2011;6:e21474.

[28] Tilstra JS, Clauson CL, Niedernhofer LJ, Robbins PD. NF-kB in Aging and Disease. Aging Dis. 2011;2:449-65.

[29] Al-Attar AM, Zari TA. Influences of crude extract of tea leaves, Camellia sinensis, on streptozotocin diabetic male albino mice. Saudi J Biol Sci. 2010;17:295-301.

[30] Vipin Goyal V, Chugh K, Agrawal Y. Association of serum glutamic pyruvic transaminase and non-alcoholic fatty liver disease in controlled and uncontrolled diabetes. J Health Spec. 2014;2:169-173.

[31] Xourafas D, Ardestani A, Ashley SW, Tavakkoli A. Impact of weight-loss surgery and diabetes status on serum ALT levels. Obes Surg. 2012;22:1540-7. 